\title{
Design of an Error-Based Adaptive Controller for a Flexible Robot Arm Using Dynamic Pole Motion Approach
}

\author{
Ki-Young Song, ${ }^{1}$ Madan M. Gupta, ${ }^{1}$ and Noriyasu Homma ${ }^{2}$ \\ ${ }^{1}$ Intelligent Systems Research Laboratory, College of Engineering, University of Saskatchewan, Saskatoon, Sk, Canada S7N5A9 \\ ${ }^{2}$ Research Division on Advanced Information Technology, Cyberscience Center, Tohoku University, Sendai 980-8579, Japan
}

Correspondence should be addressed to Madan M. Gupta, madan.gupta@usask.ca

Received 16 July 2011; Accepted 12 October 2011

Academic Editor: Ivo Bukovsky

Copyright ( $2011 \mathrm{Ki}$-Young Song et al. This is an open access article distributed under the Creative Commons Attribution License, which permits unrestricted use, distribution, and reproduction in any medium, provided the original work is properly cited.

\begin{abstract}
Design of an adaptive controller for complex dynamic systems is a big challenge faced by the researchers. In this paper, we introduce a novel concept of dynamic pole motion (DPM) for the design of an error-based adaptive controller (E-BAC). The purpose of this novel design approach is to make the system response reasonably fast with no overshoot, where the system may be time varying and nonlinear with only partially known dynamics. The E-BAC is implanted in a system as a nonlinear controller with two dominant dynamic parameters: the dynamic position feedback and the dynamic velocity feedback. For illustrating the strength of this new approach, in this paper we give an example of a flexible robot with nonlinear dynamics. In the design of this feedback adaptive controller, parameters of the controller are designed as a function of the system error. The position feedback $K_{p}(e, t)$ and the velocity feedback $K_{v}(e, t)$ are continuously varying and formulated as a function of the system error $e(t)$. This approach for formulating the adaptive controller yields a very fast response with no overshoot.
\end{abstract}

\section{Introduction}

Recently, there has been an increasing interest in the design of feedback controllers: from the design of conventional approaches to the design of intelligent-based approaches. One such approach is on the design of adaptive controller for controlling a complex dynamic system containing nonlinearity like flexible joints. During the past, there has been a common practice to approximate a nonlinear system by a linear system in limited operating ranges and then make use of the conventional controller design approaches. However, the nonlinearity of a system is inevitable since many systems in practice involve nonlinear relationships among the variables such as electromechanical systems, hydraulic systems, and pneumatic systems [1]. For decades, various schemes of adaptive control have been proposed, and adaptive control for nonlinear systems with complex dynamics has received great attention. However, not many of these approaches are suitable for complex nonlinear systems [24]. Up to the present, inverse optimal controller [5-7] using the Lyapunov function has been considered as one of the most effective way for designing controllers for nonlinear systems.

In this paper, we introduce a new notion of controller called error-based adaptive controller (E-BAC) with a novel conception based upon dynamic pole motion (DPM) approach. In general, for the design of E-BAC, we consider two dominant parameters, the position feedback $K_{p}(e, t)$ and velocity feedback $K_{v}(e, t)$, and a proper design of these two feedback parameters will yield a faster and stable response of the system with no overshoot. The feedback parameters are adapted by the system error $e(t)$ and its states $\mathbf{x}(t)$.

The rest of the paper is organized as follows. In Section 2, we introduce some important observations of a step response for a typical linear second-order system. In Section 3, we describe the notion of dynamic pole motion (DPM) and the design of error-based adaptive controller (E-BAC) in detail. A flexible robotic joint control is presented in Section 4 with E-BAC and DPM as a case study. Section 5 concludes this paper with a discussion and future works. 


\section{Some Important Observations in the Step Response for a Second-Order Linear System}

In our study, we consider a typical open-loop second-order plant $G_{p}(s)$ defined as

$$
G_{p}(s)=\frac{1}{s^{2}+a s+b},
$$

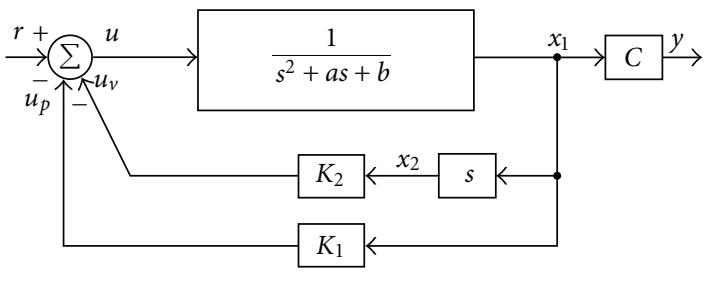

$$
\begin{aligned}
& \text { state vector: } \mathbf{x}=\left[\begin{array}{l}
x_{1} \\
x_{2}
\end{array}\right]=\left[\begin{array}{c}
\frac{1}{C} y \\
\dot{x}_{1}
\end{array}\right], \\
& \text { output: } y=C x_{1}=\left(b+K_{1}\right) x_{1} .
\end{aligned}
$$

Equations (1a), (1b), and (1c) represent a typical secondorder system with position $\left(K_{1}\right)$ and velocity $\left(K_{2}\right)$ feedbacks.

As shown in (1b), with position and velocity feedback controller, the transfer function of the closed-loop system is given by

$$
\frac{Y(s)}{R(s)}=\frac{C}{s^{2}+\left(a+K_{2}\right) s+\left(b+K_{1}\right)},
$$

where $C=b+K_{1}$. This transfer function can be compared with a general linear second-order system model as

$$
\frac{\left(b+K_{1}\right)}{s^{2}+\left(a+K_{2}\right) s+\left(b+K_{1}\right)}=\frac{\omega_{n}^{2}}{s^{2}+2 \zeta \omega_{n} s+\omega_{n}^{2}} .
$$

Thus, we see that

$$
\begin{aligned}
& \omega_{n}^{2}=\left(b+K_{1}\right) \triangleq K_{p}, \quad \omega_{n}: \text { system natural frequency, } \\
& 2 \zeta \omega_{n}=\left(a+K_{2}\right) \triangleq K_{v}, \quad \zeta: \text { system damping ratio, }
\end{aligned}
$$

where the parameters $K_{p}$ and $K_{v}$ are defined as position feedback and velocity feedback, respectively.

Generally the dynamic behavior of a second-order system can be described in terms of two dominant parameters, the natural frequency $\left(\omega_{n}\right)$ and the damping ratio $(\zeta)$. The transient response of a typical control system often exhibits damped oscillations before reaching the steady state. In specifying the transient response characteristics of a secondorder control system to a unit-step input, the following

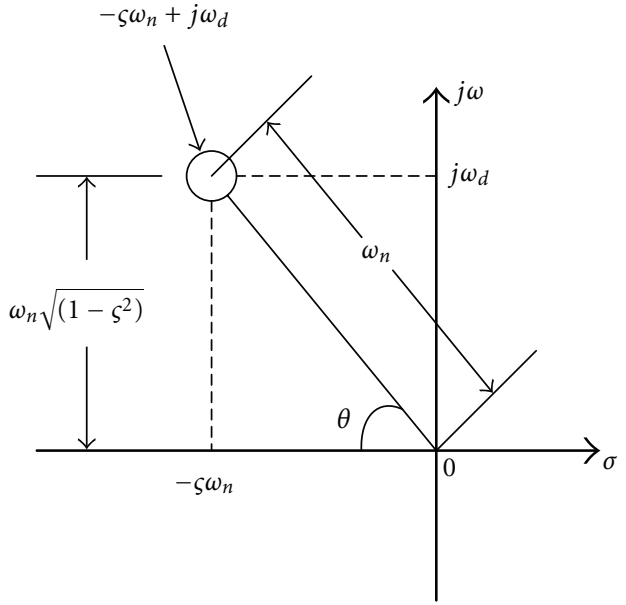

FIGURE 1: Definition of the various parameters in the complex $\sigma-j \omega$ plane: natural frequency: $\omega_{n}$, damping ratio: $\zeta$, damped natural frequency: $\omega_{d}=\omega_{n} \sqrt{\left(1-\zeta^{2}\right)}$, and $\theta=\cos ^{-1} \zeta$.

transient parameters in the design of a controller are usually considered $[1,8,9]$ :

rise time: $T_{r}=\frac{\pi-\theta}{\omega_{d}}=\frac{\pi-\theta}{\omega_{n} \sqrt{1-\zeta^{2}}}, \quad \theta=\cos ^{-1}(\zeta)$,

settling time: $T_{s}=\frac{4}{\zeta \omega_{n}} \quad(2 \%$ criterion $)$,

maximum overshoot: $M_{p}=e^{-\zeta \pi / \sqrt{1-\zeta^{2}}} \times 100 \quad(\%)$,

bandwidth: $\omega_{\mathrm{BW}}=\omega_{n} \sqrt{\left(1-2 \zeta^{2}\right)+\sqrt{4 \zeta^{4}-4 \zeta^{2}+2}}$.

It is important to note that in the step response of the secondorder system, the dominant transient parameters $T_{r}, T_{s}$, and $M_{p}$ are dependent upon the natural frequency $\left(\omega_{n}\right)$ and the damping ratio $(\zeta)$ of the system. Thus, the positions of the poles of the system are determined by the values of $\omega_{n}$ and $\zeta$ as shown in Figure 1.

As shown in Figure 2, it is also to be noted that, in typical transient responses an underdamped system $(\zeta<1)$ yields a faster rise time $\left(T_{r}\right)$ at the expense of a large overshoot $\left(M_{p}\right)$ and a large settling time $\left(T_{s}\right)$, whereas an overdamped system $(\zeta>1)$ yields no overshoot, that is, $M_{p}=0$, but it yields large $T_{r}$ and $T_{s}$.

\section{Development of an Error-Based Adaptive Controller (E-BAC): Some Design Criteria}

For the design of an appropriate feedback controller, let us consider the system error $e(t)$ as an important signal in our feedback design. In our design methodology developed in this paper, we will make the parameters of the feedback controller as functions of the error. From the transient responses shown in Figure 2(b), we can emphasize that for large errors a small $\zeta$ and a large $\omega_{n}$, (i.e., an underdamped dynamics with large bandwidth) will yield a very fast 


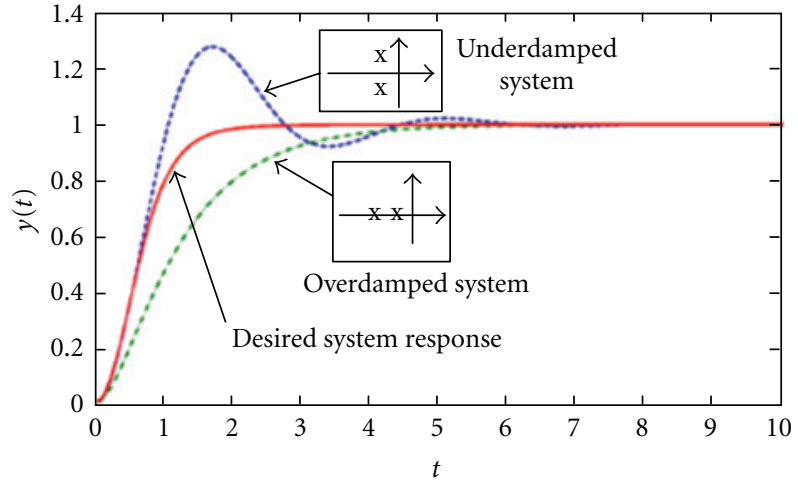

(a) The system response curves

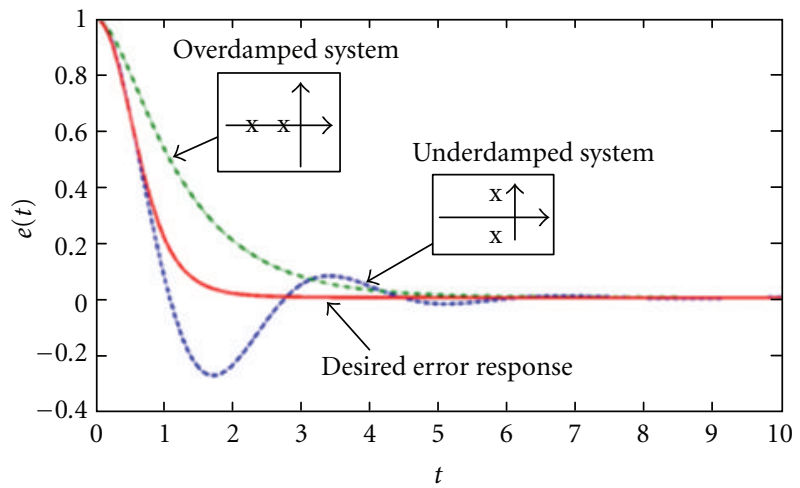

(b) The error response curves of the systems

FIGURE 2: System responses to a unit-step input with two different locations of poles (i) underdamped case $(\zeta<1)$ and (ii) overdamped case $(\zeta>1)$. The desired system response curve initially follows the underdamped curve for large errors and then settles down to a steady-state value (following the overdamped curve) for decreasing errors.

response with a very small rise time $T_{r}$. On the other hand, for small errors a large $\zeta$ and a small $\omega_{n}$ (i.e., an overdamped system with a small bandwidth) will inhibit any overshoot. Since $\zeta$ and $\omega_{n}$ are dependent upon the parameters of position feedback $\left(K_{p}\right)$ and velocity feedback $\left(K_{v}\right)$, if we define $K_{p}(e, t)$ and $K_{v}(e, t)$ as functions of the system error, $e(t)=r(t)-y(t)$, then we can achieve a very fast dynamic response with no overshoot.

From these qualitative observations on the transient response of the step response, we derive the following design criteria for the E-BAC [10].

\section{Design Criteria for the Error-Based Adaptive Controller (E-BAC)}

(i) If the system error is large, then keep the damping ratio $\zeta$ very small and natural frequency $\omega_{n}$ very large. A large $\omega_{n}$ and small $\zeta$ will result into a large bandwidth of the system, thereby a shorter rise time and fast response.

(ii) If the system error is small, then keep the damping ratio $\zeta$ large and natural frequency $\omega_{n}$ small. This will

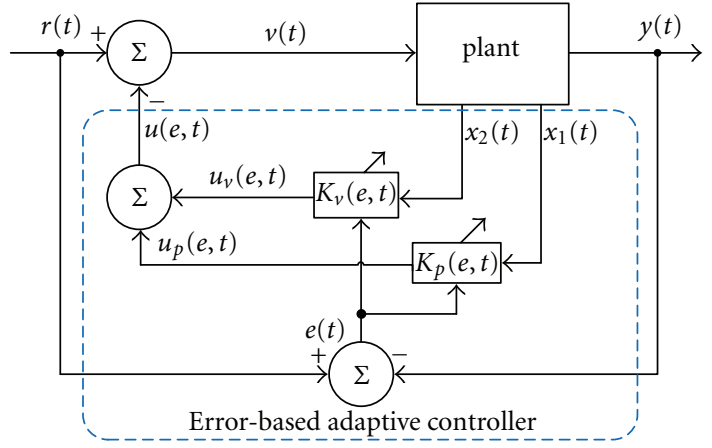

FIGURE 3: The proposed error-based adaptive controller (E-BAC): $x_{2}(t)=\dot{x}_{1}(t) . K_{p}(e, t)$ and $K_{v}(e, t)$ are defined in (8) and (12).

result into a small bandwidth of the system. For small errors, a large damping ratio in the system will avoid any overshoot in the system response.

\section{Design of Parameters for the E-BAC}

(i) Position feedback $K_{p}$ controls the natural frequency $\omega_{n},\left(K_{p}=\omega_{n}^{2}\right)$, and, therefore, the bandwidth of the system.

(ii) Velocity feedback $K_{v}$ controls the damping ratio $\zeta$, $\left(K_{v}=2 \zeta \omega_{n}\right)$.

Thus, we design the adaptive controller parameters which, in this case, are the position feedback $K_{p}(e, t)$ and velocity feedback $K_{v}(e, t)$ as functions of the error, $e(t)$. This procedure for designing the adaptive controller will introduce a dynamic motion in the poles of the system keeping the system response at an acceptable level. Here thus, we introduce a new notion of the movable poles and give it the name Dynamic Pole Motion (DPM). The proposed novel EBAC is illustrated in Figure 3.

This novel design philosophy for adaptive controller is translated into the following linguistic algorithm:

As error decreases from a large value to a small value, $K_{p}(e, t)\left(=\omega_{n}^{2}(t)\right)$ is continuously decreased from a very large value to a small value, and simultaneously, $K_{v}(e, t)\left(=2 \zeta(t) \omega_{n}(t)\right)$ is increased from a small value to a large value.

This linguistic control algorithm causes a larger bandwidth with a smaller damping ratio for large errors and smaller bandwidth with larger damping ratio for small errors. Hence, as discussed above and shown in Figures 2 and 3, during the operation of the system a desired transient response from the systems can be achieved by varying $\omega_{n}$ and $\zeta$ as functions of error. As given in (4), $\omega_{n}$ and $\zeta$ are dependent upon the position feedback $K_{p}$ and velocity feedback $K_{v}$, respectively. Some typical response curves for a second-order closed-loop system with varying $K_{p}$ and $K_{v}$ are shown in Figure 4 .

3.1. Design of E-BAC Parameters $K_{p}(e, t)$ and $K_{v}(e, t)$. Using the design criteria for the adaptive controller stated above, 


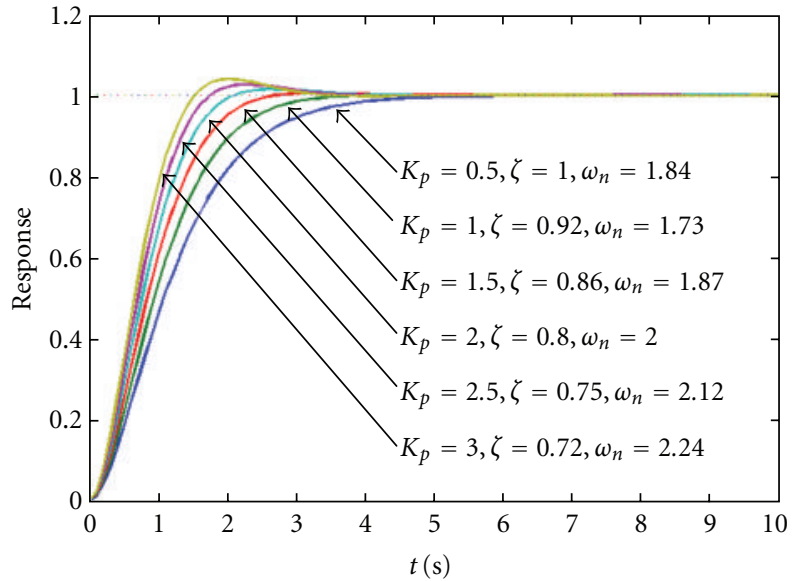

(a) A family of system response curves with various values of $K_{p}$ and a constant $K_{v}=3$

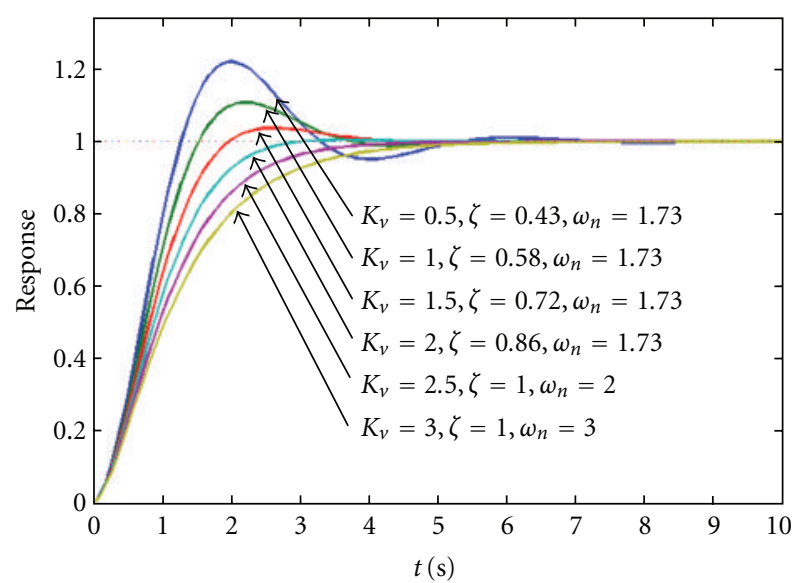

(b) A family of system response curves with various values of $K_{v}$ and a constant $K_{p}=1$

FIGURE 4: System response curves of a second-order system varying $K_{p}$ (position feedback) and $K_{v}$ (velocity feedback).

one can develop many types of functions for $K_{p}(e, t)$ and $K_{v}(e, t)$, which satisfy the design criteria with respect to the system error and time. Here, we give one such function for $K_{p}(e, t)$ and $K_{v}(e, t)$ by defining the system error as

$$
e(t)=r(t)-y(t)
$$

where the system output $y(t)$ is given by

$$
y(t)=K_{p}(e, t) x_{1}(t) .
$$

Thus, we define the position feedback $K_{p}(e, t)$ and the velocity feedback $K_{v}(e, t)$ gains as functions of $e(t)$ as

$$
\begin{gathered}
K_{p}(e, t)=K_{p f}\left(1+\alpha e^{2}(t)\right), \\
K_{v}(e, t)=K_{v f} \exp \left[-\beta e^{2}(t)\right],
\end{gathered}
$$

where $\alpha$ and $\beta$ are some gain constants which decide the slope of the functions and affect the system response (see Figure 5), $K_{p f}$ and $K_{v f}$ are the final steady-state values of
TABLE 1: Various possible functions and their graphic experessions for feedback gains $K_{p}(e, t)$ and $K_{v}(e, t)$.

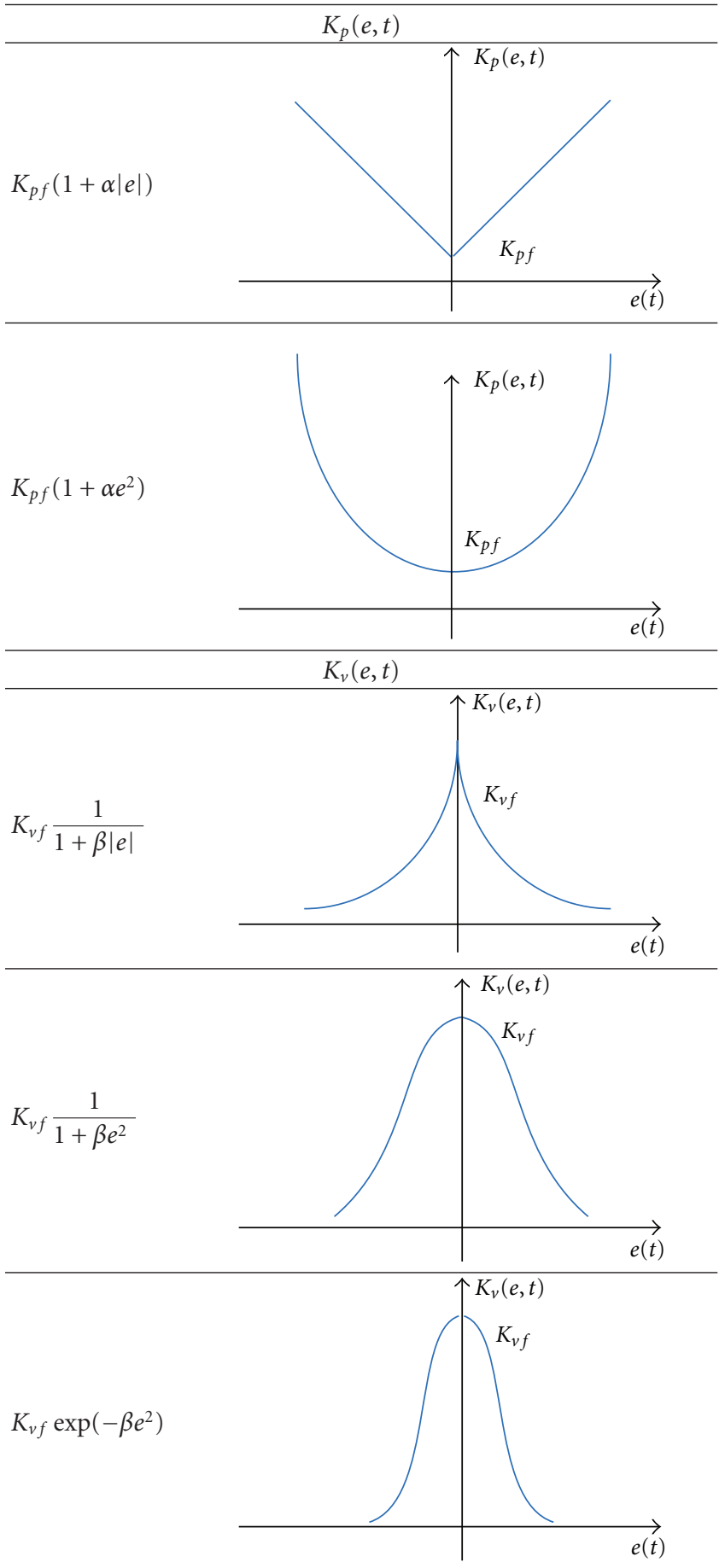

Many other functions can be derived for $K_{p}(e, t)$ and $K_{v}(e, t)$, for example, using the hyperbolic tangent and cosine functions.

$K_{p}(e, t)$ and $K_{v}(e, t)$, and $\exp (\cdot)$ is the exponential function. The other possible functions for $K_{p}(e, t)$ and $K_{v}(e, t)$ are given in Table 1.

3.2. Design of the Error-Based Adaptive Controller (E-BAC). The error-based adaptive control signal $u(e, t)$ is derived as a 


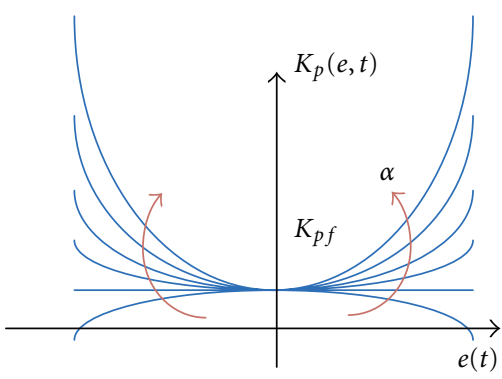

(a) Changes in slope for $K_{p}(e, t)=K_{p f}(1+$ $\left.\alpha e^{2}\right)$ for various values of $\alpha$ : the direction of arrows indicates the increasing value of $\alpha$ from negative to positive values

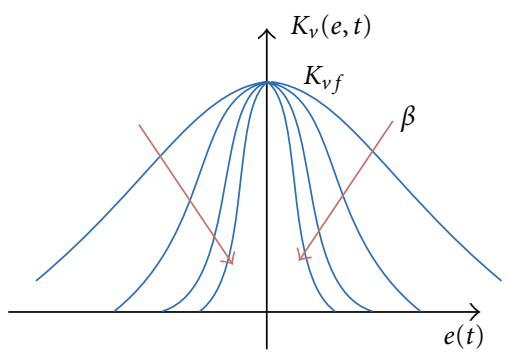

(b) Changes in slope for $K_{v}(e, t)=$ $K_{v f} \exp \left[-\beta e^{2}(t)\right]$ for various values of $\beta$ : the direction of arrows indicates the increasing value of $\beta$

FIgure 5: The change of the slopes of $K_{p}(e, t)$ and $K_{v}(e, t)$ curves for various values of $\alpha$ and $\beta$.

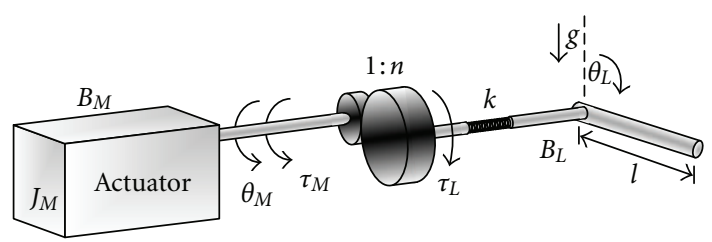

FIGURE 6: A schematic diagram of a single link manipulator with a flexible joint.

function of the error $e(t)$ and time $t$ using the following two steps:

position feedback control: $u_{p}(e, t)=K_{p}(e, t) x_{1}(t)$,

velocity feedback control: $u_{v}(e, t)=K_{v}(e, t) x_{2}(t)$,

where $K_{p}(e, t)$ and $K_{v}(e, t)$ are defined in (8) and (12), respectively. Thus, the total feedback signal $u(e, t)$ is given by

$$
u(e, t)=u_{p}(e, t)+u_{v}(e, t),
$$

and the control signal $v(t)$ (see Figure 3 ) is defined as

$$
v(t)=r(t)-u(e, t) .
$$

\section{A Case Study: Control of a Flexible Robot Arm Using E-BAC}

In this section, we present the design and simulation studies of the proposed error-based adaptive controller (E-BAC) for a flexible joint of a robot arm.

4.1. Modeling of a Single Link Flexible Robot. As shown in Figure 6, a single link manipulator with flexible joint consists of an actuator connected through a gear train (harmonic drive) with the ratio $n$ to a rigid link with length $l$, mass $m$, and moment of inertia $m l^{2} / 3$.

Let us symbolize the rotor inertia of the actuator $J_{M}$, the viscous damping of the actuator $B_{M}$, the relative angular displacement of the joint actuator $\theta_{M}$, a torque to the motor shaft $\tau_{M}$, and the relative displacement of the end effector (load) $\theta_{L}$. The joint flexibility is modeled by a linear torsional spring with stiffness $k$. The dynamics of the manipulator with a flexible joint can be represented by Euler-Lagrange equation defining $\tau_{M}=r$ as $[11,12]$

$$
\begin{gathered}
\frac{m l^{2}}{3} \ddot{\theta}_{L}+B_{L} \dot{\theta}_{L}+\frac{m g l}{2} \sin \theta_{L}+k\left(\theta_{L}+\frac{\theta_{M}}{n}\right)=0, \\
J_{M} \ddot{\theta}_{M}+B_{M} \dot{\theta}_{M}+\frac{k}{n}\left(\theta_{L}+\frac{\theta_{M}}{n}\right)=r .
\end{gathered}
$$

Equation (12) can be rewritten using the state variables $x_{i}$ $(i=1,2,3,4)$ defining as

$$
x_{1}(t)=\theta_{M}, \quad x_{2}(t)=\dot{\theta}_{M}, \quad x_{3}(t)=\theta_{L}, \quad x_{4}(t)=\dot{\theta}_{L} .
$$

Thus, we have

$$
\begin{gathered}
\dot{x}_{1}(t)=x_{2}(t), \\
\dot{x}_{2}(t)=-a_{1} x_{1}(t)-a_{2} x_{2}(t)-a_{3} x_{3}(t)+b r(t), \\
\dot{x}_{3}(t)=x_{4}, \\
\dot{x}_{4}(t)=-a_{4} x_{1}(t)-a_{5} x_{3}(t)-a_{6} \sin \left(x_{3}(t)\right)-a_{7} x_{4}(t),
\end{gathered}
$$

where

$$
\begin{aligned}
b & =\frac{1}{J_{M}}, \quad a_{1}=\frac{k}{J_{M} n^{2}}, \quad a_{2}=\frac{B_{M}}{J_{M}}, \quad a_{3}=\frac{k}{J_{M} n}, \\
a_{4} & =\frac{3 k}{m n l^{2}}, \quad a_{5}=\frac{3 k}{m l^{2}}, \quad a_{6}=\frac{3 g}{2 l}, \quad a_{7}=\frac{3 B_{L}}{m l^{2}} .
\end{aligned}
$$

The block diagram of the system is shown in Figure 7. This system is a nonlinear and time varying system since the sine function in the feedback loop of the system causes nonlinearity in the system. The output of the system is dependent on the amplitude of the control signal, and if we use the conventional design tools, this nonlinearity causes some problems in designing an effective controller. In this paper, we present a novel approach to the design of a controller for this nonlinear timevarying system by using the error-based adaptive controller (E-BAC) and dynamic pole motion (DPM). 


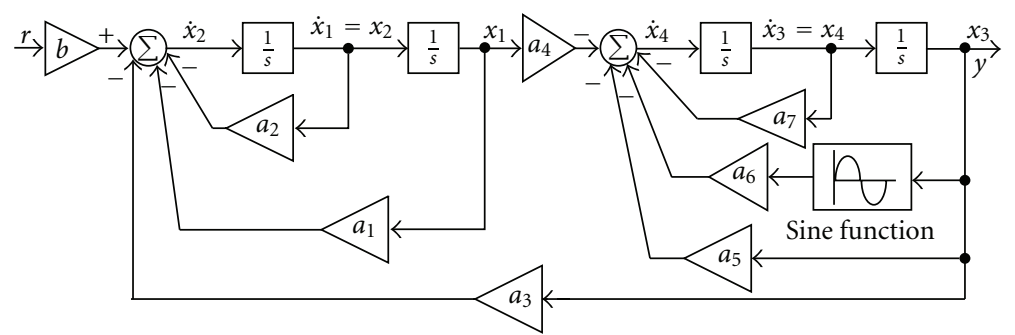

FIGURE 7: Block diagram of the single link manipulator with a flexible joint. The system has both linear and nonlinear feedbacks.

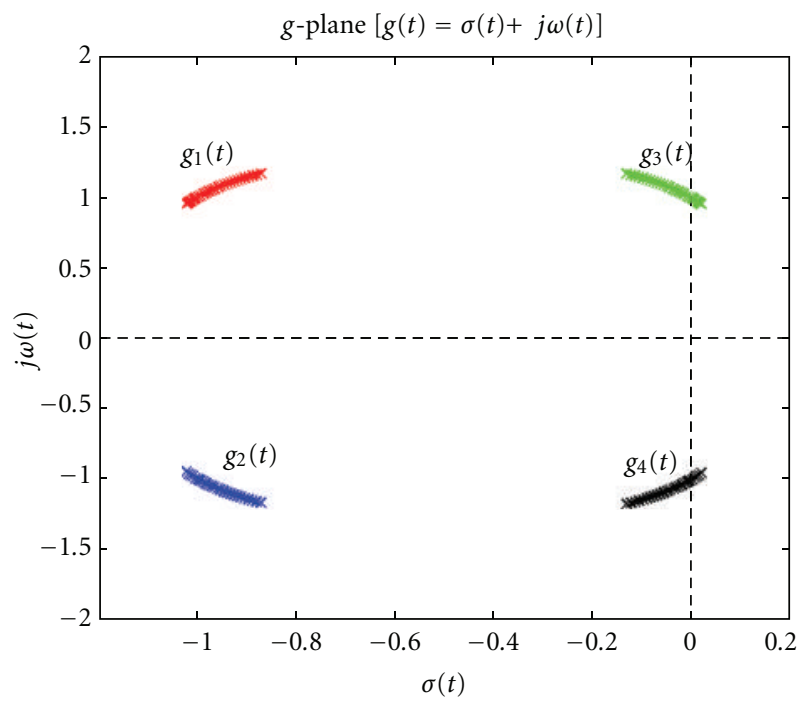

Figure 8: The sketch of dynamic pole motion (DPM) of the single link manipulator with a flexible joint system, (18), without a controller. Four poles are moving in the $g(t)=\sigma(t)+j \omega(t)$ plane with varying $x_{3}(t)$. Note that for certain values of $x_{3}(t)$ two dynamic poles move towards the right-hand side of the $g$-plane causing instability in the system.

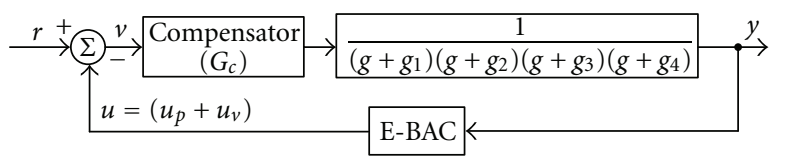

Figure 9: Schematic diagram of the flexible joint of a robot arm with a compensator $\left(G_{c}\right)$ and E-BAC.

4.2. Design of Adaptive Controller for the Systems. In this case study, for simplicity we set the value of the parameters $a_{i}(i \in$ $[1,7])$ and $b$ equal to 1 . Thus, (14) can be rewritten as

$$
\begin{gathered}
\dot{x}_{1}(t)=x_{2}(t), \\
\dot{x}_{2}(t)=-x_{1}(t)-x_{2}(t)-x_{3}(t)+r(t), \\
\dot{x}_{3}(t)=x_{4}(t), \\
\dot{x}_{4}(t)=-x_{1}(t)-\left\{1-\frac{\sin \left(x_{3}(t)\right)}{x_{3}(t)}\right\} x_{3}(t)-x_{4}(t) .
\end{gathered}
$$

Now we design an error-based adaptive controller for the single link robotic manipulator with a flexible joint. We

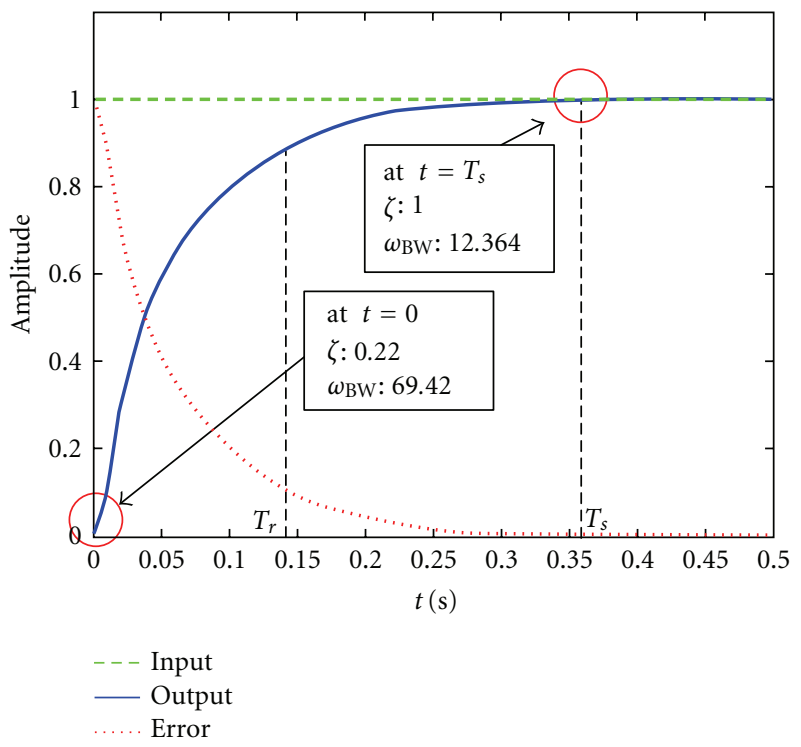

Figure 10: The system response with the E-BAC to a step reference input. Rise time $T_{r}=0.14$ seconds; settling time $T_{s}=0.36$ seconds.

first formulate the dynamic characteristic equation of the system. In this study for the nonlinear and timevarying robot arm, a new notion of timevarying complex variable $g(t)=$ $\sigma(t)+j \omega(t)(g$-plane $)$ is applied instead of the time invariant complex variable $s=\sigma+j \omega$ (s-plane). The $g$-plane has the same properties of $s$-plane with an additional property of timevarying. The dynamic characteristic equation of the single-link manipulator with a flexible joint shown in Figure 7 and described in (16) is given by

$g^{4}(t)+2 g^{3}(t)+(3+\psi(t)) g^{2}(t)+(2+\psi(t)) g(t)+2+\psi(t)=0$,

where $\psi(t)=\sin \left(x_{3}(t)\right) / x_{3}(t)$.

The dynamic roots of this characteristic equation of the transfer function can be calculated as

$$
\begin{aligned}
& g_{1,2}(t)=\frac{1}{2}\left\{-1-\sqrt{-2 \psi(t)-3 \pm 2 \sqrt{\psi^{2}(t)-4}}\right\}, \\
& g_{3,4}(t)=\frac{1}{2}\left\{-1+\sqrt{-2 \psi(t)-3 \pm 2 \sqrt{\psi^{2}(t)-4}}\right\} .
\end{aligned}
$$

The nonlinear function, $\psi(t)=\sin \left(x_{3}(t)\right) / x_{3}(t)$, covers the range $-0.22<\psi(t)<1$ for all values of $x_{3}(t)$ over $[-\infty, \infty]$. 


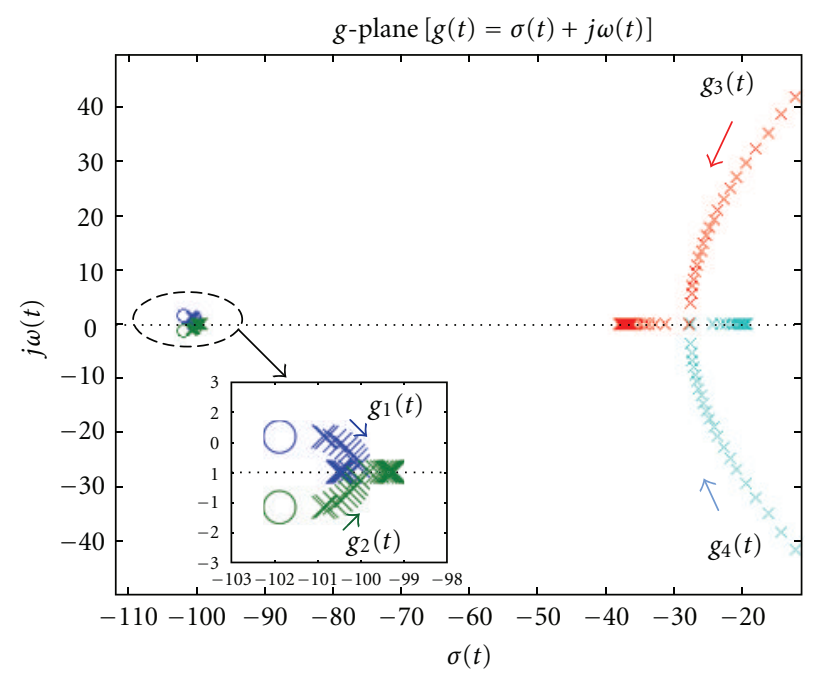

(a) The dominant poles are moving from initial position $g_{3,4}(0)=-10.24 \pm j 45.14$ to final position $g_{3}(0.5)=-37.9557$ and $g_{4}(0.5)=-19.2109$, respectively. This dynamic pole motion causes the system response to become from underdamped response to overdamped response

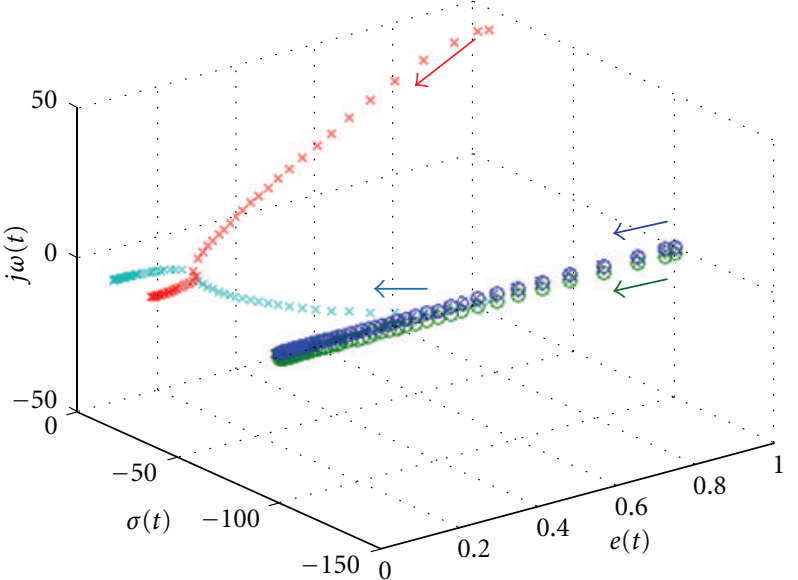

(b) 3D sketch of the dynamic poles and zeros motion of the controlled system. The location of the dynamic poles and zeros is a function of the system error

FIGURE 11: The sketch of motions of dynamic poles and zeros of the system with a compensator and an E-BAC ( $\times$ : poles, $\bigcirc$ : zeros).

Thus, the roots of the dynamic characteristic equation are moving in the $g$-plane $(g(t)=\sigma(t)+j \omega(t))$.

The moving roots of the dynamic characteristic equation are named as dynamic poles. (Note that in linear timeinvariant dynamic systems since the parameters of the system remain constant, the poles and zeros of the system are time invariant.) The plot of the four dynamic poles of this flexible robot arm without a controller is shown in Figure 8. From this figure, it is clear that for some values of $x_{3}$ two dynamic poles move towards the right-hand side (RHS) of the $g(t)=\sigma(t)+j \omega(t)$ plane causing instability in the system. The design criteria of our proposed error-based adaptive controller (E-BAC) for a system are as below.

Design Criteria of E-BAC for the Flexible Joint of a Robot Arm. For designing an E-BAC, we should consider the following important points.

(1) For introducing the stability in the robot arm system, we should move the dynamic poles on the left-hand side (LHS) on $g(t)=\sigma(t)+j \omega(t)$ plane for all values of $x_{3}(t)$.

(2) Realization of DPM using E-BAC.

(a) For achieving the fast response time, the system must have a large bandwidth for large errors and small bandwidth for small errors. Thus, the position feedback $K_{p}$, the bandwidth parameter, must be a function of the system error $e(t)$.

(b) For no overshoot in the system response, damping should be adjusted continuously as a function of the system error. The position feedback $K_{p}(e, t)$ and the velocity feedback
$K_{v}(e, t)$ are designed such that they yield a small damping ratio with large bandwidth for large errors and a large damping ratio with small bandwidth for small errors.

For achieving a good controller for this fourth-order flexible robot arm, we must first add a compensator to relocate two dynamic poles far away from the origin in the left-half of the $g$-plane. In this case study, we relocate $g_{1}(t)$ and $g_{2}(t)$ far away from the $j \omega(t)$-axis. These two relocated poles far away in the left-side of the $g$-plane will induce very small time constants, thereby, will have negligible effect in the system dynamic response. The other two poles that are closer to the imaginary axis are dominant poles and will cause an influence in the system dynamic response. Then, an E-BAC is added in the feedback loop with a position feedback $K_{p}(e, t)$ and a velocity feedback $K_{v}(e, t)$ defined in the previous sections. In this study, the compensator $\left(G_{c}\right)$ used introduces two zeros in the forward loop with the position of zeros being $g(t)=-102 \pm$ j1.18. This compensator provides a control over the plant poles $\left[g_{1}(t)\right.$ and $\left.g_{2}(t)\right]$ keeping them far away from the $j \omega(t)$ axis. The feedback controller, E-BAC, provides a control over the two plant poles $\left[g_{3}(t)\right.$ and $\left.g_{4}(t)\right]$. The diagram of the system with a compensator and an E-BAC is illustrated in Figure 9.

The control input signal $v(t)$ is derived using (11) as

$$
v(t)=r(t)-u(t),
$$

where

$$
\begin{gathered}
u(t)=\left\{u_{p}(e, t)+u_{v}(e, t)\right\}, \\
u_{p}(e, t)=K_{p f}\left[1+\alpha e^{2}(t)\right] x_{3}(t), \\
u_{v}(e, t)=\exp \left[-\beta e^{2}(t)\right] x_{4}(t) .
\end{gathered}
$$




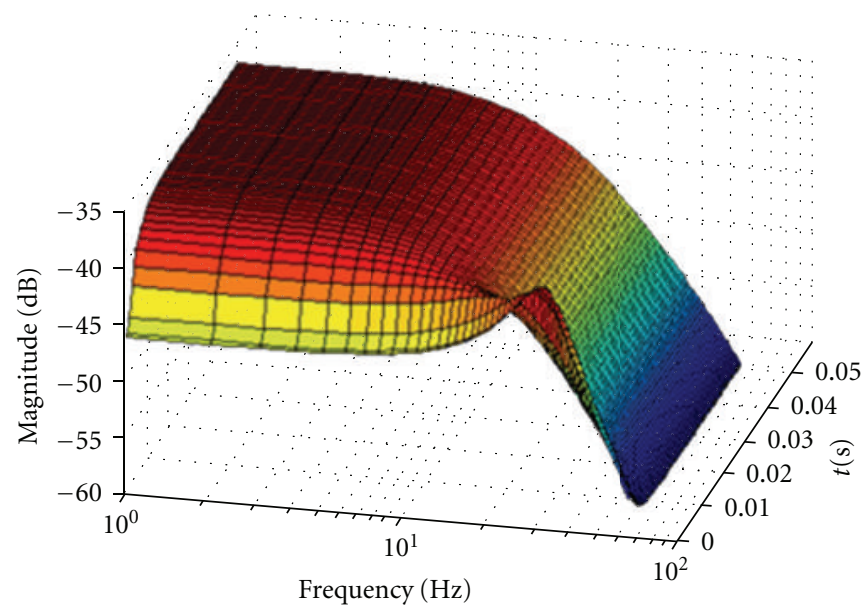

(a) Bandwidth, $\omega_{\mathrm{BW}}(t)$

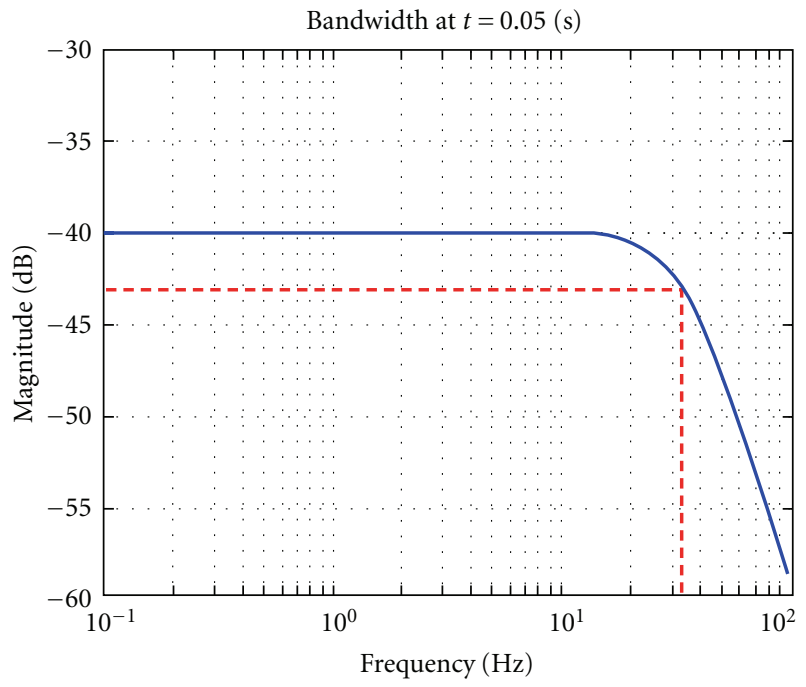

(c) $\omega_{\mathrm{BW}}(0.05) \approx 32 \mathrm{~Hz}$

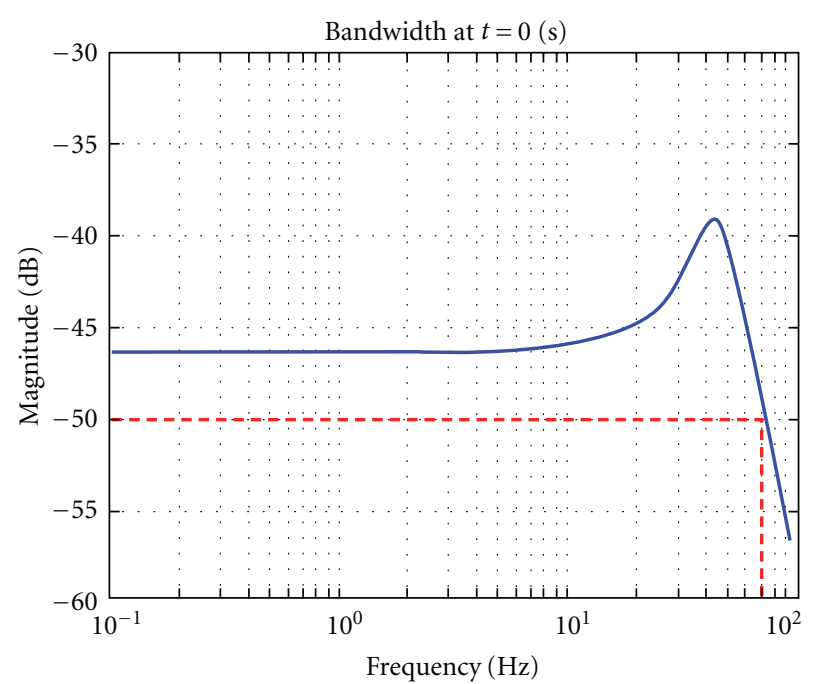

(b) $\omega_{\mathrm{BW}}(0) \approx 70 \mathrm{~Hz}$

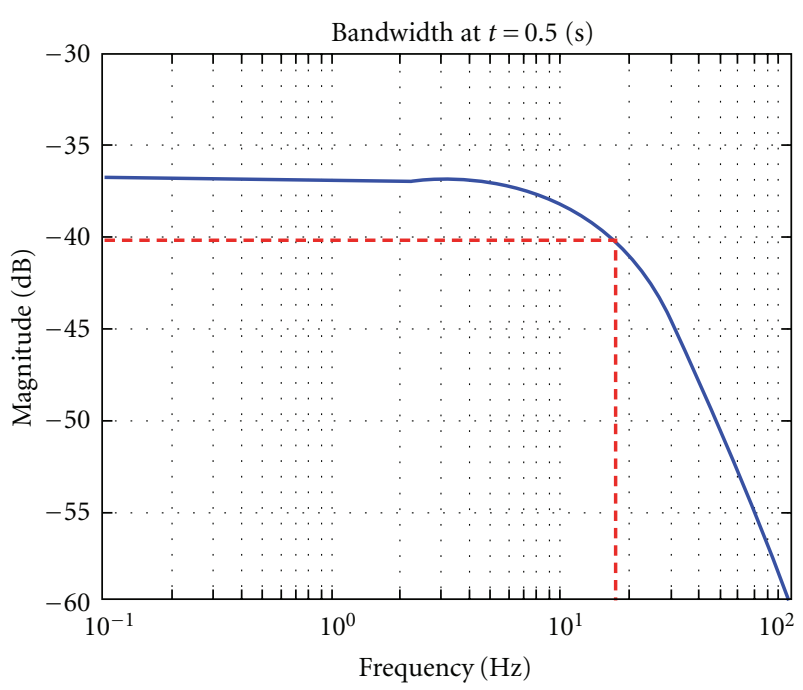

(d) $\omega_{\mathrm{BW}}(0.5) \approx 18 \mathrm{~Hz}$

FIGURE 12: The variation of the bandwidth of the controlled system. (a) 3D sketch of the variation of the bandwidth with respect to the frequency at each time interval, (b) the bandwidth at $t=0(\mathrm{sec})$, (c) the bandwidth at $t=0.05(\mathrm{sec})$, and (d) the bandwidth at $t=0.5$ (sec).

$x_{3}(t)=x$ and $x_{4}(t)=\dot{x}$ are the states of the system, $K_{p f}$ and $K_{v f}$ are the steady-state values of feedbacks $K_{p}(e, t)$ and $K_{v}(e, t)$, respectively, $\alpha$ and $\beta$ are some gain constants for $K_{p}(e, t)$ and $K_{v}(e, t)$, respectively, $r(t)$ is the reference input of the system, and $e(t)=[y(t)-r(t)]=\left(r(t)-K_{p}(e, t) x_{3}(t)\right)$ is the system error.

As described in the design criteria, the objective of the embedded E-BAC is to design the control $u(t)$ to make the system output $y(t)$ follow the reference input signal $r(t)$ as closely as possible with fast rise time $T_{r}$ and small settling time $T_{s}$ with no overshoot $M_{p}$. Thus, we continuously change the dynamics of the close-loop system: initially for large errors, we make large bandwidth and very small damping ratio $\zeta(t)$, and as error decreases, the damping ratio $\zeta(t)$ is continuously increased and the system bandwidth is decreased.
In the design of the E-BAC, we have arbitrarily chosen the gains $\alpha=2, \beta=1, K_{p f}=70$, and $K_{v f}=5.5$. With these values, the controlled system responded as an underdamped system for large error at $t=0$, which continuously moved towards an overdamped system with decreasing error.

4.3. Simulation Results. Using the gains $\alpha=2, \beta=1, K_{p f}=$ 70 , and $K_{v f}=5.5$, the initial positions of the dynamic poles of the system are placed at $g_{1,2}(0)=-100.87 \pm j 1.18$ (relocated to far from $j \omega(t)$-axis by the compensator), and $g_{3,4}(0)=-10.24 \pm j 45.1$. During the operation of the system, as error is decreased to zero, the final positions of the dynamic poles are moved to $-100.5639,-99.2695$, -37.9557 , and -19.2109 on the $g$-plane. The zeros are located at around -100 near the relocated poles by the compensator, and the zeros attract the relocated poles not 


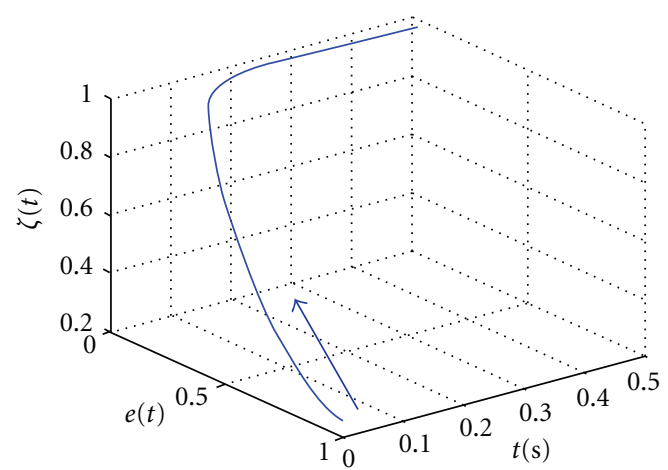

FIgURE 13: The variation of the damping ratio $\zeta(t)$ with respect to the system error as a function of time. The value of $\zeta(t)$ changes from a low value, $\zeta(t)=0.221$, to a high value, $\zeta(t)=1$.

to affect the dominant poles. The results of the simulation study of this case study are shown in Figure 10. Further, the maps of the dynamic poles motion (DPM) are illustrated in Figure 11. The output response initially follows the trajectory with a large bandwidth and a small damping ratio, which settles down with a large damping and a smaller bandwidth.

It is clear from the figure that the dynamic motion of poles of the system is decided by the value of the system error. The initial positions of the dominant dynamic poles are placed to generate a low damping ratio $\zeta(t)$ and large bandwidth of the system. Thus, initially the system is underdamped. Thereafter, the dynamic poles are optimized and shifted as the system error decreases reducing the bandwidth and increasing $\zeta(t)$. The final positions of the dominant dynamic poles make the system an overdamped system. Thus, the bandwidth becomes small, but $\zeta(t)$ becomes high. The variation of the bandwidth at each time interval is shown in Figure 12, and the variation of the damping ratio $\zeta(t)$ is shown with respect to the system error at each time interval in Figure 13.

\section{Discussion and Conclusions}

In this paper, we have proposed the design of an error-based adaptive controller (E-BAC) for controlling the dynamic response of a nonlinear system. The proposed E-BAC is the controller with continuously changing feedback parameters as functions of the system error: initially for large errors an underdamped system with large bandwidth which is forced to approach to become an overdamped system with small bandwidth for small errors. In the beginning, for large errors the system is underdamped, thus, it makes the system faster with a wider bandwidth. As the error decreases, the value of the feedback gains $K_{p}$ decreases and $K_{v}$ increases. The design of this adaptive controller is conceptually error-based and can be used to handle the complexity of systems. In order to support the novel controller, we introduce the notion of dynamic pole motion (DPM).

As a case study, we present a flexible joint of robot arm, which is a nonlinear dynamic system, and this system is controlled by the proposed E-BAC. Without a proper controller, the system is unstable due to the nonlinearity in the feedback loop of the system. However, as shown in Figure 10, with E-BAC the trajectory response of the system is very fast, $T_{r}=0.36$ seconds, without any overshoot $\left(M_{p}=0 \%\right)$. Also, as shown in Figures 12 and 13, in this step response, the initial bandwidth of the system is very high $(\approx 70 \mathrm{~Hz})$ which settles down to about $18 \mathrm{~Hz}$ in the steadystate situation. The bandwidth of the system changes from a large value to a small value. Similarly but contrarily, the damping ratio $\zeta(t)$ varies from $0.221(t=0)$ to $1(t=0.5)$. From the simulation studies, it is shown that the proposed E-BAC is able to control nonlinear time varying systems. Conventionally, a proper design of the controller guarantees that the changing pole position is always positioned in the left-hand side (LHS) on $g$-plane. In this novel design approach, the dynamic poles are always located in LHS on $g$-plane, thus the stability of the controlled system is assured. Further work is under way to extend this E-BAC design philosophy for higher-order partially known and unknown complex dynamic systems.

\section{References}

[1] K. Ogata, Modern Control Engineering, Prentice Hall, Upper Saddle River, NJ, USA, 4th edition, 2002.

[2] Y. C. Chang, "An adaptive $\mathrm{H} \infty$ tracking control for a class of nonlinear multiple-input-multiple-output (MIMO) systems," IEEE Transactions on Automatic Control, vol. 46, no. 9, pp. 1432-1437, 2001.

[3] D. G. Taylor, P. V. Kokotovic, R. Marino, and I. Kanellakopoulos, "Adaptive regulation of nonlinear systems with unmodeled dynamics," IEEE Transactions on Automatic Control, vol. 34, no. 4, pp. 405-412, 1989.

[4] Y. Liu and X. Y. Li, "Robust adaptive control of nonlinear systems represented by input-output models," IEEE Transactions on Automatic Control, vol. 48, no. 6, pp. 1041-1045, 2003.

[5] M. Jankovic, R. Sepulchre, and P. V. Kokotovic, "Global adaptive stabilization of cascade nonlinear systems," Automatica, vol. 33, no. 2, pp. 263-268, 1997.

[6] R. Sepulchre, M. Jankovic, and P. V. Kokotovic, "Integrator forwarding: a new recursive nonlinear robust design," Automatica, vol. 33, no. 5, pp. 979-984, 1997.

[7] E. D. Sontag, "A 'universal' construction of Artstein's theorem on nonlinear stabilization," Systems and Control Letters, vol. 13, no. 2, pp. 117-123, 1989.

[8] N. S. Nise, Control Systems Engineering, John Wiley \& Sons, New York, NY, USA, 3rd edition, 2000.

[9] W. J. Palm III, System Dynamics, McGraw-Hill, New York, NY, USA, 2005.

[10] K. Y. Song, M. M. Gupta, D. Jena, and B. Subudhi, "Design of a robust neuro-controller for complex dynamic systems," in Proceedings of the Annual Meeting of the North American Fuzzy Information Processing Society (NAFIPS '09), Cincinnati, Ohio, USA, June 2009.

[11] M. W. Spong, K. Khorasani, and P. V. Kokotovic, "An integral manifold approach to the feedback-control of flexible joint robots," IEEE Journal of Robotics and Automation, vol. 3, no. 4, pp. 291-300, 1987.

[12] L. Jin, M. M. Gupta, and P. N. Nikiforuk, "Dynamic recurrent neural networks for modeling flexible robot dynamics," in Proceedings of the 10th IEEE International Symposium on Intelligent Control, pp. 105-110, August 1995. 

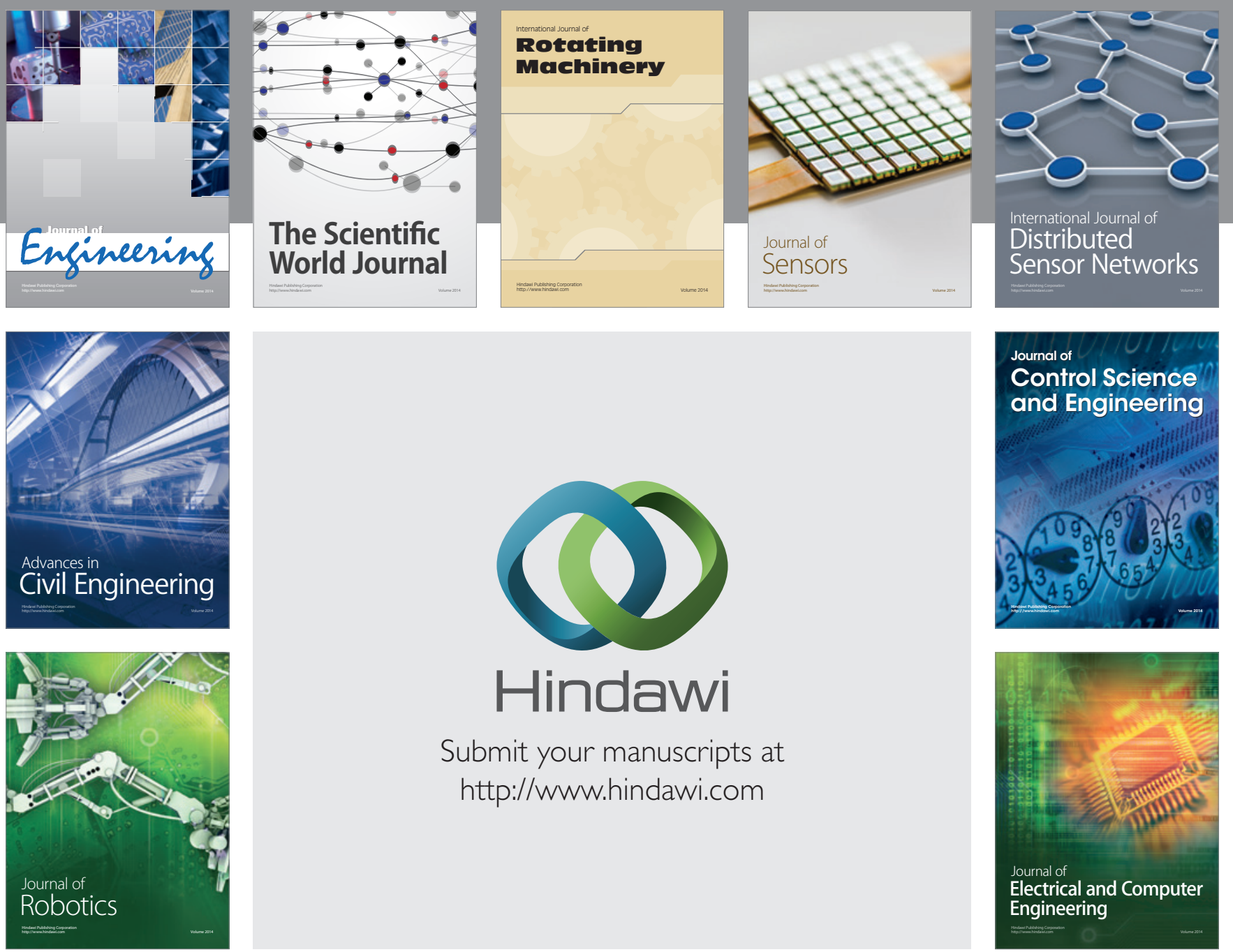

Submit your manuscripts at

http://www.hindawi.com
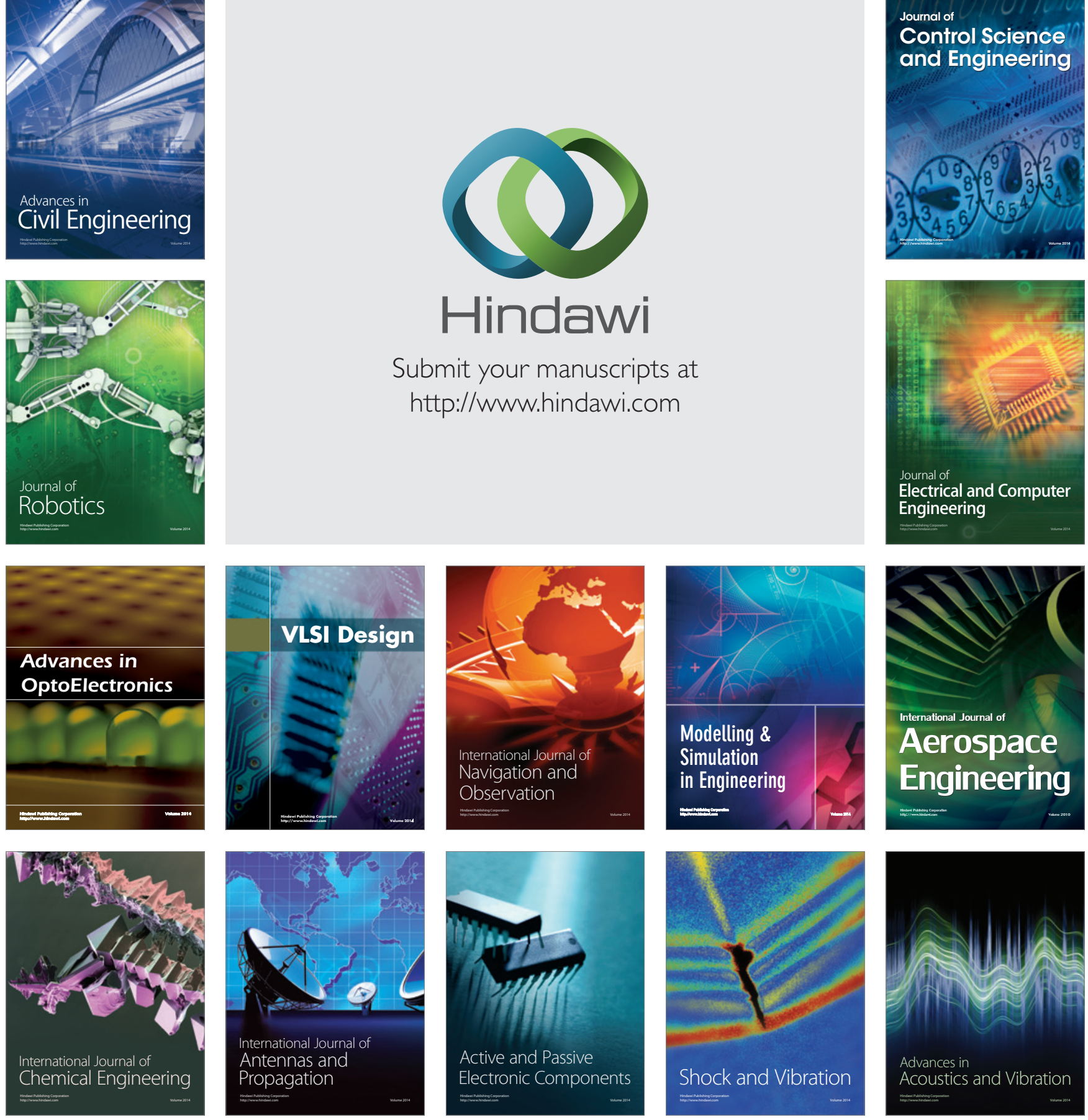